\title{
Comparative Study on Chinese Characters between Mainland Chinese Handwriting and Taiwanese Handwriting
}

\author{
Yuchen Wang ${ }^{1}$, Zhongyuan $\mathbf{J i}^{1}$ \\ ${ }^{1}$ Key Laboratory of Evidence Identification in Universities of Shandong Province, Shandong University of \\ Political Science and Law, Jinan, Shandong Province, China
}

Keywords: Chinese handwriting; compare; writing rules

\begin{abstract}
With cross-straits exchanges and cooperation constantly deepened, there will be more and more Taiwanese writer identification cases in judicial appraisals. But in the field of handwriting identification of mainland, there is little study of Taiwanese handwriting, no more for a comparative study on Chinese characters between Mainland Chinese handwriting and Taiwanese handwriting. This paper analyzes the Chinese characters of Taiwanese handwriting and the differences between Mainland Chinese handwriting and Taiwanese handwriting, which can provide some suggestions for the actual case.
\end{abstract}

\section{Introduction}

With the in-depth development of reform and opening up, the exchanges between the mainland and Taiwan have become more and more frequent. As a common language for cross-strait exchanges, Chinese characters are widely involved in daily life and judicial practice may involve more cases of Taiwanese handwriting identification. At present, there is no research on the relevance of Taiwanese Chinese characters in the mainland. Although the Chinese character system of Taiwan is homologous to the Chinese character system of the mainland, due to the development of history, it is still affected by the Roman, Japanese, Taiwanese and Chinese characters and there are still some differences with the Chinese character system. This inevitably causes the differences of the Chinese characters between Taiwanese handwriting and mainland Chinese handwriting.

\section{Comparisons of Experimental Samples}

For the convenience of discussion, this paper compares the cross-strait experimental samples according to the feature classifications of the mainland handwriting inspection.

\subsection{Overview of handwriting features.}

Font features. The 50 Taiwanese samples are mainly free-styled Xingkai (Figure 1). The 50 mainland samples are also mainly free-styled Xingkai (Figure 2).

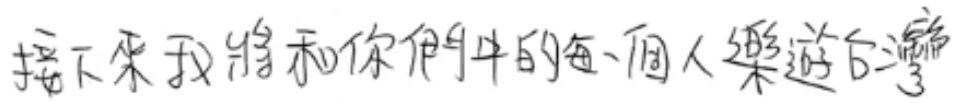

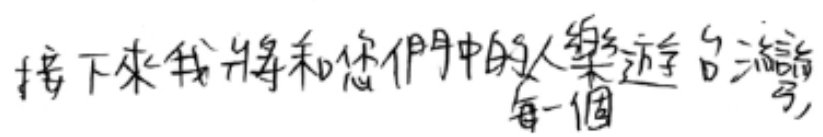

Figure 1 Taiwanese samples

$$
\begin{aligned}
& \text { 接下来或将和保们中的每一个人可, 游占涪 } \\
& \text { 接下来我将和你们中的娒个人业游台棺 }
\end{aligned}
$$

Figure 2 Mainland samples 
Word size features. Among the 50 samples from Taiwan, 18 of them have larger size of single word $\left(\geq 1 \mathrm{~cm}^{*} 1 \mathrm{~cm}\right)$ while the other 32 samples are medium-sized $\left(0.5 \mathrm{~cm}^{*} 0.5 \mathrm{~cm}-1 \mathrm{~cm}^{*} 1 \mathrm{~cm}\right)$. Among the 50 mainland samples, only 3 of them have larger size of single word $(\geq 1 \mathrm{~cm} * 1 \mathrm{~cm})$ while the other 47 samples are medium-sized $\left(0.5 \mathrm{~cm}^{*} 0.5 \mathrm{~cm}-1 \mathrm{~cm}^{*} 1 \mathrm{~cm}\right)$.

Comparing the cross-strait samples, the reason why more Taiwanese samples are larger is that the strokes of a traditional Chinese character in Taiwan is more. In the case of A4 paper without grid lines, when the size of the single word space is not limited, it is easy to cause a large single word in order to clearly write each stroke.

Overall layout features. Text direction and word direction: The 50 Taiwan samples are written from left to right and from the top to the bottom in the overall layout text direction, as well as its word direction. The 50 continent samples are also moved from left to right and from the top to the bottom in the overall layout of the text direction and the word direction.

Comparing the cross-strait samples, there is no vertical handwriting from the top to the bottom and from the right to the left of the Taiwanese samples. It may be because Taiwan has generally entered the horizontal printing system and the collection of samples is limited.

\subsection{Features of partial arrangement.}

Some of the 50 Taiwan samples indent at the beginning of the segment while the others not and the formulaic sequences are also relatively random. The 50 continental samples are more random at the position of each beginning segment and the formulaic sequences.

\subsection{Writing features of Chinese characters.}

Writing features of traditional Chinese characters. There are a total of 167 Chinese characters in dictation materials. There are more traditional Chinese characters in the 50 Taiwan handwriting samples. The least are 46 traditional fonts and the most are 50 traditional characters (Figure 3). However, the 50 mainland samples are written in the current simplified Chinese characters without any traditional characters (Figure 4).

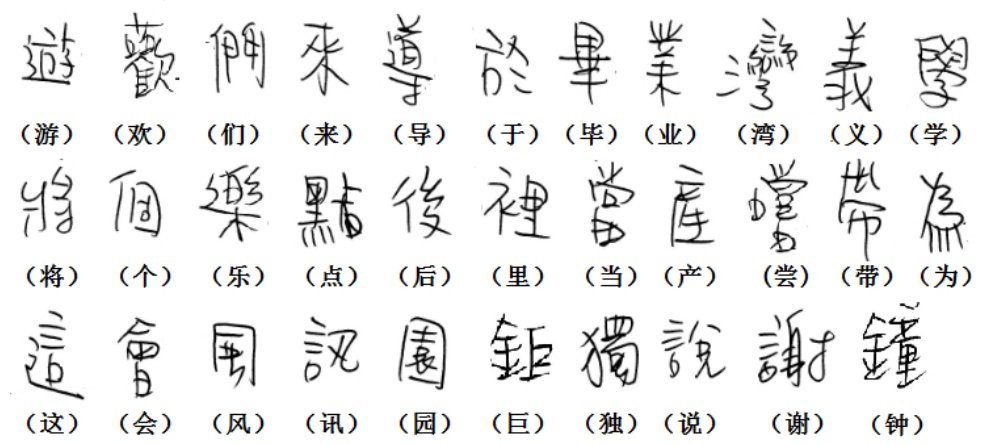

Figure 3 Traditional Chinese characters in Taiwan sample

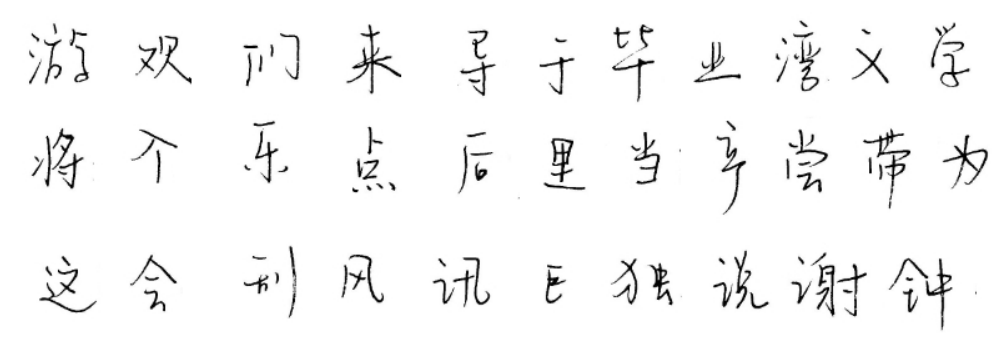

Figure 4 Simplified Chinese characters in mainland samples

Multiple ways of handwriting. In the Taiwan sample, there are many ways to write "点(dian), 刮 (gua), 尝(chang)" (Figure 5). Among the 50 Taiwanese samples of "点(dian)", 34 are written in the traditional Chinese form of "點(dian)"; 11 are written in the variant form of "占(dian)"; only 5 are written in the siplified form of "点(dian)" which is the same handwriting with the mainland samples. Among the 50 Taiwanese samples of "刮(gua)",18 are written in the traditional Chinese form of "颭 
(gua)"; 12 are written in the form of "括(gua)" which is the homophone of "刮(gua)"; 20 are written in the siplified form of "刮(gua)" which is the same handwriting with the mainland samples. Among the 50 Taiwanese samples of "尝(chang)", 22 are written in the traditional Chinese form of "嘗(chang)"; 28 are written in the variant form of "嚐(chang)". In the 50 mainland samples, all the handwriting of " 点(dian), 刮(gua), 尝(chang)" are the current simplified Chinese characters.

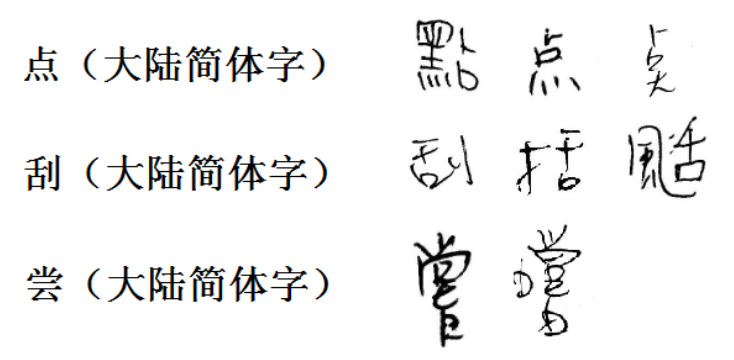

Figure 5 Multiple Taiwanese handwriting of "点(dian), 刮(gua), 尝(chang)"

\subsection{Features of the typos.}

In the 50 Taiwanese samples of "湾(wan)", four of them are different from the others (Figure 6) while the other 46 are all in the traditional Chinese character form of "灣(wan)". Among the four samples, one of them used the pinyin "Taiwan" instead of writing "台湾(Taiwan)"; one of them used "弯(wan)" instead of writing "湾(wan)"; the other two had an across between the upper and lower right sides of the "湾(wan)".

The 50 mainland samples are all the current simplified form of "湾(wan)".

\begin{tabular}{|c|c|}
\hline Taina.n & Tainan \\
\hline 立紊 & 紊 \\
\hline$u_{b}=\frac{\dot{m}}{3}$ & $\tilde{b}=\frac{\bar{u}_{k}}{3}$ \\
\hline 分 涪 & 公丵 \\
\hline
\end{tabular}

Figure 6 Taiwanese samples

Since the dictation material used UHF Chinese characters, there were no typos in the 50 mainland samples. Taiwanese simplification does not simplify the writing of "灣(wan)" into "湾(wan)" and the traditional Chinese character "灣(wan)" has many strokes. In view of the frequent cross-strait exchanges, the mainland's simplified word "湾(wan)" has been accepted by some people in Taiwan. However, some people have insufficient understanding of the simplified word "湾(wan)" and cause the mistakes.

\subsection{Features of matching proportion.}

The Taiwanese sample has certain differences from the mainland samples in the matching proportion of the words "巨(ju)", "瓦(wa)", "鼎(ding)".

Differences in the matching proportion of "巨(ju)". The 50 Taiwanese samples are all written in the traditional Chinese character form, "鉅(ju)". While writing the stroke of " $\llcorner ", 40$ of them are clearly divided into two broken strokes of " | " and "- " (Figure 7). 10 of them are wrote in one stroke. The 50 mainland samples are all written in one stroke. None of them is divided into two strokes (Figure 8). 


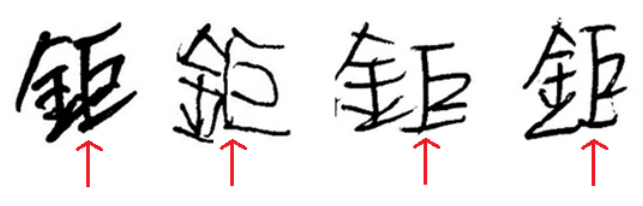

Figure 7 Taiwanese samples

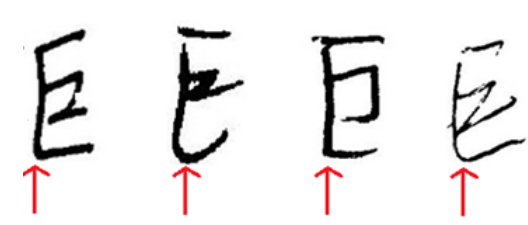

Figure 8 Mainland samples

Differences in the matching proportion of "瓦(wa)". While writing the strokes of " $L$ ", 33 of the 50 Taiwanese samples are clearly divided into two strokes while the other 17 are wrote in one stroke (Figure 11). The 50 mainland samples are all written in one stroke. None of them is divided into two strokes (Figure 12).

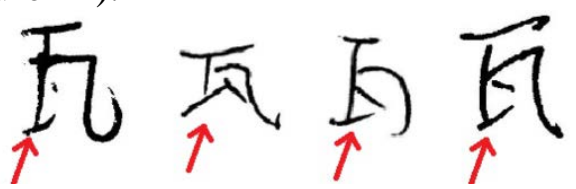

Figure 11 Taiwanese samples
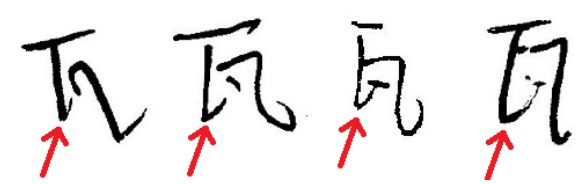

Figure 12 Mainland samples

Differences in the matching proportion of "鼎(ding)". While writing the stroke of "', 20 of them are clearly divided into two strokes of " $\llcorner$ " and " | " while the other 30 are wrote in one stroke (Figure 13). The 50 mainland samples are all written in one stroke. None of them is divided into two strokes (Figure 14).

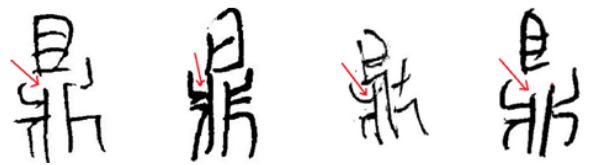

Figure 13 Taiwanese samples

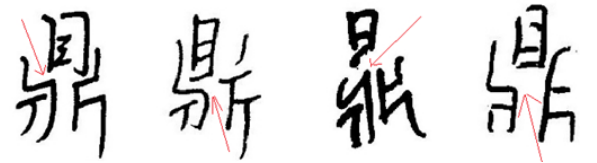

Figure 14 Mainland samples

\subsection{Features of stroke order.}

The stroke orders of "巨(ju)" are different among the Taiwanese and mainland samples. The part of "巨(ju)" both in the Taiwanese and mainland samples is chosen to compare with each other.

There are three types of stroke orders in the 50 Taiwanese samples (Figure 15). 40 of them adopt the first stroke order; 5 of them adopt the second stroke order; 4 of them adopt the third stroke order; the other one cannot be judged. There are two kinds of stroke orders in the 50 mainland samples (Figure 16). 26 of them adopt the first stroke order; 20 of them adopt the second stroke order; the other four cannot be judged. Comparing the cross-strait samples, the first stroke order of Taiwanese samples is frequent because it is common in Taiwan's writing specification, writing the stroke of " $\llcorner$ " into two strokes, which is different from the mainland standard stroke order or the universal stroke order.

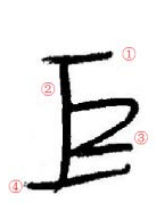

Figure 15 Taiwanese samples

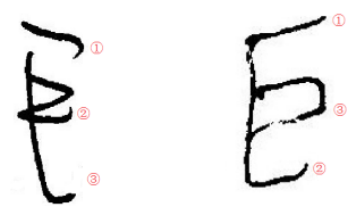

Figure 16 Mainland samples

\subsection{Features of wielding the pen.}

Three words of "户(hu)", "雨(yu)" and "说(shuo)" in the Taiwanese samples are different from those of the mainland samples in wielding the pen.

The differences of wielding the pen of "户(hu)". There are three types of "房(fang)" in the 50 mainland samples, all from left to right (Figure 17). There are four types of "房(fang)" in the 50 Taiwanese samples and two of them are from left to right (Figure 18). Because "戶(hu)" is a traditional Chinese character in Taiwan and its first stroke is " J ". The writing difference causes the 
differences of wielding the pen. 40 of the Taiwanese samples are from right to left in wielding the pen.

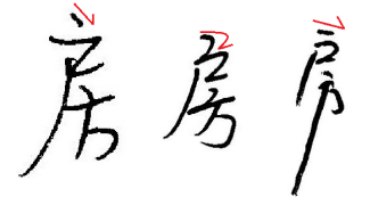

Figure 17 Mainland samples

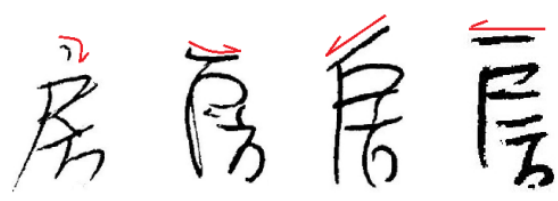

Figure 18 Taiwanese samples

The differences of wielding the pen of "雨(yu)". In wielding the pen of " 、 " in the word of "雨 (yu)", the mainland samples have two types: one is parallel and the other is cross (Figure 19). 42 of them are in parallel while the other 8 are in cross. In the Taiwanese samples, there is only one cross type (Figure 20), which is caused by the writing ways of Taiwan's traditional Chinese characters.

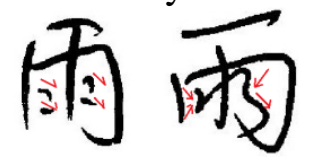

Figure 19 Mainland samples

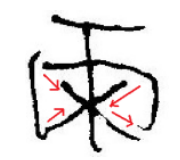

Figure 20 Taiwanese samples

The differences of wielding the pen of "说(shuo)". In the 50 mainland samples, the part of "\" in the word "说(shuo)" are crossed at the below (Figure 21); while in the 50 Taiwanese samples, the part of "\" in the word "说(shuo)" are crossed in the top (Figure 22). This is caused by the writing ways of Taiwan's traditional Chinese characters.

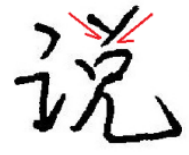

Figure 21 Mainland samples

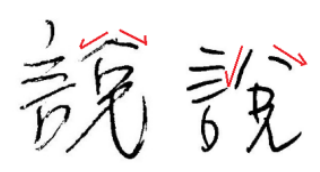

Figure 22 Taiwanese samples

\section{Conclusion}

Taiwan has two ways in text direction and word direction, that is, the horizontal writing from top to bottom and the vertical writing from right to left. Due to the influence of Taiwan's education system, in the primary and secondary school students' homework of "national language" (Chinese), the vertical writing from right to left is more frequently used.

Taiwanese often adopt the traditional Chinese characters in writing. And the phenomenon of mixing traditional Chinese characters with simplified Chinese characters is more common. When there is no grid limit, the single character is easy to be too larger and there are fewer high-level writers when writing texts.

Some commonly used simplified characters in Taiwanese handwriting are prone to mixed use of multiple writing ways. Some Chinese characters are prone to differences in matching proportion and stroke order due to the different cross-strait writing specifications.

While writing some simplified Chinese characters commonly used in cross-strait exchanges (the current simplified Chinese characters that are not found in Taiwan's writing norms), it is easy for Taiwanese to have typos due to the limited understanding.

\section{References}

[1] J. W. Chen. Chinese Character Simplification after the Founding of the People's Republic of China [J]. Beijing Archives. 2009 (04): 101.

[2] Y. Z. Zhang. Document Identification [M]. Taiwan: Angle, 2010: 85-89.

[3] Y. W. Jia, M. L. Zou. China Criminal Science and Technology Encyclopedia Document Inspection [M]. Beijing: Publishing House of Chinese People's Public Security University, 2002: 68. 
[4] J. T. Huang. Document Identification [M]. Beijing: Publishing House of Chinese People's Public Security University, 2003: 59-79. 